\title{
Mapping of the internal capsule with subcortical stimulation for gross-total resection of a thalamic metastatic tumor
}

\begin{abstract}
Sima Sayyahmelli, MD, ${ }^{1}$ Ilhan Aydin, MD, ${ }^{1}$ Bryan Wheeler, ${ }^{2}$ and Mustafa K. Baskaya, MD1
'Department of Neurological Surgery, University of Wisconsin-Madison School of Medicine and Public Health; and ${ }^{2}$ Intraoperative Neuromonitoring Program Coordinator, University of Wisconsin Hospital and Clinics, Madison, Wisconsin

Although the surgical treatment of thalamic tumors remains challenging due to the proximity to the internal capsule, safe resection of gliomas or metastatic tumors of the thalamus are possible in some selected cases due to a better understanding of microsurgical anatomy and due to advances in neurophysiological mapping and monitoring.

In this video, the authors demonstrate the use of mapping of the internal capsule with direct subcortical stimulation for the resection of a metastatic tumor. The patient is a 58-year-old man with a history of renal cell carcinoma and metastasis in the left thalamus and parieto-occipital region. He underwent stereotactic radiation of both tumors at an outside hospital. Due to the increased size of both tumors and surrounding vasogenic edema, he was referred to the authors for resection. He underwent gross-total resection via an interhemispheric transcallosal approach. His postoperative course was uneventful and did not have any focal neurological deficits, including motor, sensory, or visual functions.

The authors' surgical approach to this metastatic thalamic tumor and the intraoperative real-time direct subcortical stimulation of the internal capsule during surgery are demonstrated in this video.
\end{abstract}

The video can be found here: https://youtu.be/DmDxjJUSZWU.

KEYWORDS direct subcortical stimulation; interhemispheric transcallosal approach; internal capsule; metastatic tumor; thalamic tumor; video 